\title{
Uric acid albumin ratio as a predictive marker of short-term mortality in patients with acute kidney injury
}

\section{Yasemin Özgür', Seydahmet Akın', Nuran Gamze Yılmaz', Murat Gücün ${ }^{2}$, Özcan Keskin ${ }^{1}$}

'Department of Internal Medicine, Dr. Lutfi Kırdar Kartal Training and Research Hospital, University of Health Sciences, Istanbul, Turkey

2Department of Nephrology, Kartal Koşuyolu High Speciality Educational and Research Hospital, University of Health Sciences, Istanbul, Turkey

Objective We aimed to investigate uric acid and albumin ratio (UA/A) as a marker of short-term mortality in acute kidney injury (AKI). Both uric acid and albumin are strongly correlated with the development and mortality of AKI.

Methods The patients hospitalized from May 2019 to September 2019 for AKI were included in this study. The diagnostic odds ratio (DOR), Youden index (J), and the area under a receiver operating characteristic curve (AUROC) determined a cut-off UA/A ratio for mortality. Cox-regression analysis was performed to identify UA/A as a prognostic marker of the 30-day mortality rate.

Results A total of 171 patients with an average age of $69.20 \pm 13.0$ (45.6\% women) were included in the study. The average UA/A ratio was $3.3 \pm 1.5 \mathrm{mg} / \mathrm{g}$ and $2.5 \pm 1.0 \mathrm{mg} / \mathrm{g}$ in the nonsurvivor and survivor groups, respectively $(P=0.001)$. The best cut-off $U A / A$ ratio associated with mortality was determined as $2.4 \mathrm{mg} / \mathrm{g}$ with a specificity of $52 \%$ and a sensitivity of $77 \%$ (DOR, 3.6 ; J, 28.8; AUROC, 0.644). Thirty-day cumulative survival rates of the low and high UA/A ratio groups were $85.9 \pm 4.0 \%$ and $63.7 \pm 5.0 \%$, respectively. The estimated survival times of the low and high UA/A ratio groups were 27.7 days (95\% confidence interval $[\mathrm{Cl}, 26.2-29.3)$ and 23.9 days $(95 \% \mathrm{Cl}, 22.0-25.9)$, respectively.

Conclusion We found a direct correlation between 30-day mortality and UA/A ratio at initial presentation in AKI patients regardless of age, comorbidities, and clinical and laboratory findings, including albuminuria.

Keywords Acute kidney injury; Uric acid; Serum albumin; Hypoalbuminemia; Hyperuricemia

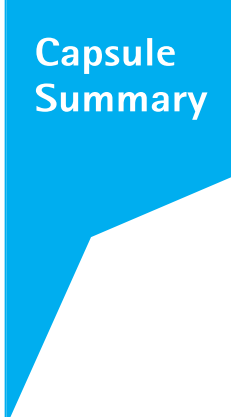

What is already known

Hypoalbuminemia and hyperuricemia have been associated with acute kidney injury and mortality separately.

What is new in the current study

We found that the relationship between 30-day mortality and uric acid albumin ratio at the time of the first presentation in patients with acute kidney injury was independent of age, comorbidities, and other clinical and laboratory findings, including albuminuria.
eISSN: 2383-4625

Received: 14 March 2020

Revised: 1 June 2020

Accepted: 30 July 2020

Correspondence to: Yasemin Özgür Department of Internal Medicine, Dr. Lutfi Kırdar Kartal Training and Research Hospital, University of Health Sciences, E-5 Highway Cevizli, 34890 Kartal, Istanbul, Turkey

E-mail: yasemin.ozgur@saglik.gov.tr ORCID

https://orcid.org/0000-0002-7112-4575

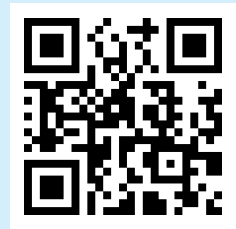

How to cite this article:

Özgür Y, Akın S, Yılmaz NG, Gücün M, Keskin Ö. Uric acid albumin ratio as a predictive marker of short-term mortality in patients with acute kidney injury. Clin Exp Emerg Med 2021;8(2):82-88. https://doi. org/10.15441/ceem.20.024

This is an Open Access article distributed under the terms of the Creative Commons Attribution Non-Commercial License (https:// creativecommons.org/licenses/by-nc/4.0/). 


\section{INTRODUCTION}

In addition to being the main determinant of intravascular oncotic pressure, albumin is a key molecule in the body's defense mechanisms playing roles in anti-inflammation, anti-apoptosis, and protection from oxidative stress. ${ }^{1,2}$ Aside from these important functions, it acts as a carrier of many hormones, drugs, bioactive elements, free fatty acids, calcium, iron, and bilirubin. ${ }^{3}$ Serum albumin levels may decrease due to accelerated metabolism, malnutrition, malabsorption, decreased synthesis in the liver, or capillary leakage secondary to inflammation. ${ }^{4}$ Hypoalbuminemia, which is frequently seen in chronic diseases such as heart failure, nephrotic syndrome, and cirrhosis, has been associated with prolonged hospitalization and increased mortality. ${ }^{5}$

Uric acid is a notable waste product of purine metabolism and is mostly cleared by the kidneys with a small percentage removed directly by the intestines. ${ }^{6}$ Chronic diseases such as hypertension, diabetes, chronic kidney disease, obesity, and gout as well as some drugs, especially diuretics, can increase uric acid levels in the body. ${ }^{7}$ In recent years, hyperuricemia has also been linked to increased mortality risks. ${ }^{8}$

Acute kidney injury (AKI) is diagnosed via a rapid increase in serum creatinine level, decrease in urine output, or combination of both, and is characterized by a rapid deterioration in kidney function. ${ }^{9}$ Hypoalbuminemia and hyperuricemia have been associated with AKI and result in increased mortality. ${ }^{10,11}$ According to Yeter et al., ${ }^{12}$ Uric acid albumin ratio (UA/A) higher than 1.7 was significantly associated with $\mathrm{AKI}$ and 28-day mortality. In this study, we investigate the relationship between UA/A ratio and short-term mortality in patients who have AKI.

\section{METHODS}

\section{Study design}

This study was conducted in accordance with the Declaration of Helsinki after obtaining the necessary ethics committee permission (2019/514/148/4). It was planned for in-hospital implementation. It was designed as a prospective observational study on patients with AKI by obtaining written informed consent. All patients who applied to the emergency service over the age of 18 between 1 May and 30 September 2019 who developed AKI and were seen at the internal medicine clinic for follow-up and treatment were included in the study. Patients who did not meet AKI criteria were excluded from the study. No assistance was received from any funding agency or sponsor in this study.

\section{Patient population}

According to the Kidney Disease Improving Global Outcomes (KDIGO) guide, AKI was diagnosed by meeting at least one of the following criteria: $\geq 0.3 \mathrm{mg} / \mathrm{dL}$ increase in serum creatinine level within 48 hours, greater than or equal to 1.5 times increase in serum creatinine level in the last 7 days compared to baseline, and urine output of $<0.5 \mathrm{~mL} / \mathrm{kg} / \mathrm{hr}$ in 6 hours. $^{13}$

AKI staging is also done as follows according to the KDIGO guide $^{13}$ : stage 1 , serum creatinine level increases 1.5 to 1.9 times or $\geq 0.3 \mathrm{mg} / \mathrm{dL}$ from baseline or urine output is $<0.5 \mathrm{~mL} / \mathrm{kg} / \mathrm{hr}$ for 6 to 12 hours; stage 2, serum creatinine level increased by 2.0 to 2.9 times from baseline or urine output is $<0.5 \mathrm{~mL} / \mathrm{kg} / \mathrm{hr}$ for $\geq 12$ hours; and stage 3 , serum creatinine level 3 -fold increase from baseline or $>4.0 \mathrm{mg} / \mathrm{dL}$ or requiring renal replacement therapy initiation or urine output is $<0.3 \mathrm{~mL} / \mathrm{kg} / \mathrm{hr}$ or anuric for $\geq 12$ hours.

\section{Clinical outcomes}

The socio-demographic characteristics, comorbidities, and clinical findings of all patients participating in the study were recorded in study specific report forms. In addition, length of hospital stay was recorded from the emergency admission to the discharge from the hospital. Within 30 days after the initial form completion, the status of the patients was checked through the national death notification system and was recorded in report forms. Serum creatinine (if available, also base level serum creatinine levels in the last 3 months), urea, albumin, sodium, potassium, chloride, calcium, phosphorus, uric acid, hemoglobin levels, power of hydrogen, and bicarbonate levels (the last two via venous blood gas) at the time of emergency service stay were recorded. In addition to the proteinuria, hematuria, or pyuria observed in a standard urinalysis measured with a urine dipstick; albumin level was also measured by turbidimetric method in spot urine $(\mathrm{mg} / \mathrm{L})$. Blood creatinine and spot urine creatinine $(\mathrm{mg} / \mathrm{dL})$ concentration were measured by Beckman Coulter AU5832 device (Beckman Coulter, Brea, CA USA) with kinetic color test (Jaffe method). Afterwards, spot urine albumin and creatinine levels were proportioned to each other, and urine albumin creatinine ratio was calculated in $\mathrm{mg} / \mathrm{g}$ value. Hypoalbuminemia was defined as plasma albumin level of less than $3.5 \mathrm{~g} / \mathrm{dL}$; hyperuricemia was defined as plasma uric acid level higher than $7 \mathrm{mg} / \mathrm{dL}$.

The hospital admission dates, length of hospital stay, release from the clinic dates, discharge status (as are, with cure, transfer to intensive care unit [ICU], and exit), and dates of transfer to ICU for all the patients were recorded in the same manner. The hospital stay (internal medicine ward \pm ICU) starting on the date of admission to the emergency department was recorded with the 30- 
day follow-up performed after. The in-hospital mortality status of the patients was checked from the hospital data systems; and the 30-day mortality status was checked from the national death notification system. All findings were recorded in the case report forms.

\section{Statistical analysis}

After evaluating the distributions via the Kolmogorov-Smirnov test, variables with normal distribution were expressed as mean \pm standard deviation, and those without normal distribution were expressed as median (interquartile range). Categorical variables were reported as numbers and percentages. Patient characteristics among each group were compared using the chi-squared test or Fisher exact test for categorical variables and the MannWhitney U-test or student t-test for continuous variables. To determine the best UA/A cut off level in the 30-day mortality estimate, diagnostic odds ratio (DOR; (+) likelihood ratios/(-) likelihood ratio, Youden index (J; \% sensitivity+\% specificity-100), and area under the receiver operating characteristic curve (AUROC; [sensitivity+specificity] / 2) were used. The 30-day survival analysis was performed utilizing Kaplan-Meier curves. Cox-regression analysis was utilized to identify the UA/A for mortality. P-values of $<0.05$ were considered statistically significant. All statistical analyses were performed using IBM SPSS Statistics ver. 22 (IBM Corp., Armonk, NY, USA).

\section{RESULTS}

A total of 171 patients, with an average age of $69.2 \pm 13.0$ years, $45.6 \%$ of whom were females, were seen at the internal medicine clinic in the 4 months observed, were included in the study. Among them, $62.6 \%$ of the patients were hypoalbuminemic, and $61.7 \%$ were hyperuricemic. Regarding comorbidities, $63.7 \%$ of patients had hypertension, 51.5\% had chronic kidney disease (CKD), $35.7 \%$ had diabetes mellitus (DM), 31\% had malignancy, 24.6\% had coronary artery disease, $19.3 \%$ had congestive heart failure, 14\% had chronic obstructive pulmonary disease, 9.4\% of them had a history of stroke, 5.8\% had Alzheimer disease, and 3.5\% had liver cirrhosis. The median Charlson comorbidity score of all patients was 5 (range, 0 to 14).

It was observed that $25.1 \%$ of patients (43/171) died within the 30-day period, of which 22.8\% (39/171) expired during the hospital stay. Table 1 presents the clinical features of patients grouped according to their survival. Those who were nonsurvivors were found to have had higher overall averages in age, respiratory rate, corrected calcium level, uric acid, and Charlson comorbidity score as well as higher incidences of malignancy, ascites, ede-
Table 1. Comparison of the clinical features of the patients according to their 30-day mortality

\begin{tabular}{|c|c|c|c|}
\hline & $\begin{array}{l}\text { Survivors } \\
(n=128)\end{array}$ & $\begin{array}{c}\text { Non-survivors } \\
\quad(n=43)\end{array}$ & P-value \\
\hline \multicolumn{4}{|l|}{ Demographics and comorbidities } \\
\hline Age $(\mathrm{yr})$ & $67.8(13.7)$ & $73.3(9.9)$ & 0.018 \\
\hline Sex, female & $58(45.3)$ & $20(46.5)$ & 0.891 \\
\hline Diabetes mellitus & $46(35.9)$ & $15(34.9)$ & 0.901 \\
\hline Hypertension & $80(62.5)$ & $29(67.4)$ & 0.560 \\
\hline Chronic kidney disease & $67(52.3)$ & $21(48.8)$ & 0.691 \\
\hline Liver cirrhosis & $3(2.3)$ & $3(7.0)$ & 0.168 \\
\hline Malignancy & $34(26.6)$ & $19(44.2)$ & 0.031 \\
\hline Charlson comorbidity score & $5.0(2.4)$ & $6.3(2.7)$ & 0.002 \\
\hline \multicolumn{4}{|l|}{ Clinical and laboratory findings } \\
\hline $\mathrm{SBP}(\mathrm{mmHg})$ & $123.6(27.8)$ & $116.0(29.0)$ & 0.134 \\
\hline $\mathrm{DBP}(\mathrm{mmHg})$ & $72.6(14.5)$ & $67.6(14.0)$ & 0.056 \\
\hline Pulse rate (bpm/min) & $86.3(16.6)$ & $91.2(21.0)$ & 0.130 \\
\hline Respiratory rate (rpm/min) & $14.9(4.1)$ & $19.1(6.4)$ & 0.001 \\
\hline Oxygen saturation (\%) & $95.4(2.5)$ & $94.3(4.2)$ & 0.257 \\
\hline Ascites (\%) & $13(11.6)$ & $12(30.0)$ & 0.007 \\
\hline Pretibial edema (\%) & $44(34.4)$ & $21(48.8)$ & 0.025 \\
\hline Glucose (mg/dL) & $143.0(84.8)$ & $149.8(121.0)$ & 0.691 \\
\hline Basal creatinine $(\mathrm{mg} / \mathrm{dL})$ & $1.5(0.9)$ & $1.6(1.2)$ & 0.780 \\
\hline Creatinine (mg/dL) & $4.2(1.9)$ & $3.9(2.9)$ & 0.450 \\
\hline Urea (mg/dL) & $162.2(73.5)$ & $171.5(70.7)$ & 0.469 \\
\hline Total protein (mg/dL) & $62.7(8.7)$ & $61.9(14.2)$ & 0.714 \\
\hline Albumin (mg/dL) & $33.2(6.6)$ & $29.7(6.4)$ & 0.003 \\
\hline Uric acid (mg/dL) & $8.0(2.7)$ & $9.3(4.0)$ & 0.016 \\
\hline UA/A ratio $(\mathrm{mg} / \mathrm{g})$ & $2.52(1.0)$ & $3.25(1.5)$ & 0.001 \\
\hline UACR $(\mathrm{mg} / \mathrm{g})$ & $899.8(777.7)$ & $772.4(1,480.1)$ & 0.772 \\
\hline Sodium (mEq/L) & $133.9(6.4)$ & $133.0(9.4)$ & 0.566 \\
\hline Potassium (mEq/L) & $4.9(1.1)$ & $5.0(1.1)$ & 0.068 \\
\hline Chlorine (mEq/L) & $102.1(7.5)$ & $100.4(8.6)$ & 0.237 \\
\hline Calcium (mg/dL) & $8.5(1.2)$ & $9.0(2.3)$ & 0.244 \\
\hline Corrected calcium (mg/dL) & $9.1(1.2)$ & $9.8(2.2)$ & 0.050 \\
\hline Phosphorusm (mg/dL) & $4.4(1.5)$ & $4.7(1.7)$ & 0.335 \\
\hline Hemoglobin (g/dL) & $10.0(2.4)$ & $9.6(2.4)$ & 0.334 \\
\hline Power of hydrogen & $7.32(0.1)$ & $7.34(0.1)$ & 0.045 \\
\hline Bicarbonate $(\mathrm{mEq} / \mathrm{L})$ & $18.6(4.9)$ & $19.8(5.1)$ & 0.174 \\
\hline Proteinuria (dipstick, \%) & $75(58.6)$ & $16(65.1)$ & 0.030 \\
\hline Hematuria (dipstick, \%) & $77(60.1)$ & $12(44.1)$ & 0.082 \\
\hline Leucocyturia (dipstick, \%) & $43(33.6)$ & $21(25.6)$ & 0.691 \\
\hline Hypoalbuminemia (\%) & $74(57.8)$ & $33(76.7)$ & 0.019 \\
\hline Hyperuricemia (\%) & $68(57.1)$ & $32(74.4)$ & 0.033 \\
\hline High UA/A ratio (\%) & $58(48.7)$ & $33(76.7)$ & 0.002 \\
\hline \multicolumn{4}{|l|}{ Length of hospital stay } \\
\hline In internal medicine ward without ICU & $13.7(11.4)$ & $6.8(5.8)$ & $<0.001$ \\
\hline In internal medicine ward with ICU & $14.3(12.1)$ & $11.5(8.4)$ & 0.097 \\
\hline 30-Day survival time & $30.0(0)$ & $13.5(8.9)$ & $<0.001$ \\
\hline
\end{tabular}

Values are presented as median (interquartile range) or number (\%). $\mathrm{SBP}$, systolic blood pressure; DBP, diastolic blood pressure; UA/A, uric acid albumin ratio; $U A C R$, urine albumin creatinine ratio; ICU, intensive care unit. 
ma and proteinuria with lower albumin levels. Hypoalbuminemia rates in the non-survivors and survivor groups were $76.7 \%$ and $57.8 \%$, respectively $(P=0.019)$. Hyperuricemia rates were $74.4 \%$ and $57.1 \%$, respectively $(P=0.033)$. Thus, calculated $U A / A$ ratios were higher in the non-survivor group than in the survivor group ( $76.7 \%$ and $48.7 \%$, respectively; $P=0.002$ ). Length of hospital stay, excluding ICU stays, were $6.8 \pm 5.8$ and $13.7 \pm 11.4$ days for the nonsurvivors and survivor groups $(\mathrm{P}<0.001)$; length of hospital stay including ICU were $11.5 \pm 8.4$ and $14.3 \pm 12.1$ days in the same order $(P=0.097)$.

\section{UA/A ratio as mortality indicator}

UA/A ratio was significantly higher in the non-survivors group compared to the survivor group (mean of $3.25 \pm 1.5$ and $2.52 \pm 1$, respectively; $P=0.001$ ). $R O C$ analysis was performed to determine the best $U A / A$ ratio cut-off point in mortality estimation with the best cut-off level determined at $2.36 \mathrm{mg} / \mathrm{g}$ with $52 \%$ specificity and 77\% sensitivity (AUROC, 0.644; DOR, 3.59; J, 28.8).

As seen in Fig. 1, the UA/A ratio above 2.36 in the Kaplan-Meier graphs was associated with a significant increase in the 30day mortality rate (log rank, 0.002). The 30-day cumulative survival rate of the group with low UA/A ratio was $85.9 \pm 4 \%$ and was $63.7 \pm 5 \%$ for the group with high UA/A ratio. Lastly, the estimated survival time of the group with low UA/A ratio was 27.7 days (95\% confidential interval [CI], 26.2 to 29.3) and the group with a high UA/A ratio has an estimated survival time of 23.9 days (95\% $\mathrm{Cl}, 22$ to 25.9).

By univariate cox regression analysis: age, presence of malignancy, proteinuria, Charlson comorbidity score, respiratory rate, corrected calcium, albumin, uric acid levels, and UA/A ratio were

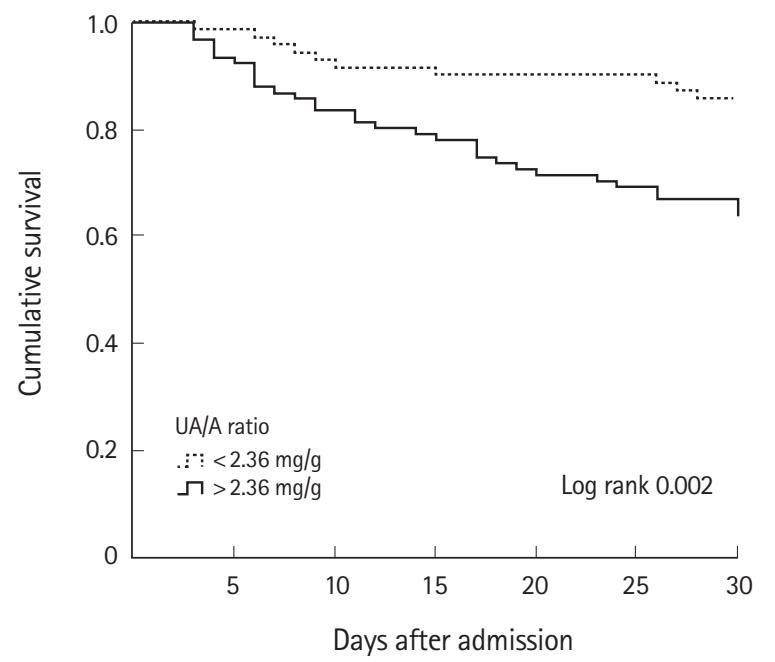

Fig. 1. Kaplan-Meier survival graphics by uric acid albumin ratio (UA/A) ratio. all related to 30-day mortality. In multivariate analysis, it was found that 30-day mortality was associated with UA/A ratio even when controlling for all the other above mentioned variables (hazard ratio, 1.39; 95\% Cl, 1.02 to $1.91 ; \mathrm{P}=0.04$ ) (Table 2).

\section{Comparative characteristics of patients according to UA/A ratio}

After determining the UA/A ratio level most associated with mortality, the patients were divided into two groups labeled as low and high UA/A ratio ( $>2.36$ and $<2.36$, respectively), and their clinical and biochemical properties were compared as seen in Table 3. Notably, there was no difference between the frequencies or duration of $\mathrm{DM}$, hypertension, $\mathrm{CKD}$, or malignancy, while those with high UA/A ratios have higher rates of liver cirrhosis and higher Charlson comorbidity scores than those with a lower UA/A ratio. When the clinical features were examined, those with high UA/A ratios had lower blood pressure and higher incidences of ascites and pretibial edema. In regards to biochemical parameters, urea and uric acid levels were found higher while albumin, sodium, and chlorine levels were found to be lower in those with high compared with those with low UA/A ratio. There was no statistically significant difference between serum total protein levels and urine albumin creatinine ratio levels between the groups. While there was no difference between the groups regarding the length of hospital stay; those with high UA/A ratio had shorter 30-day survival times ( $24 \pm 9.6$ vs. $27.7 \pm 6.6$ days), higher in-hospital mortality rates (31.9\% vs. $14.1 \%)$, and higher monthly mortality rates (36.3\% vs. $14.1 \%$ ) compared to those with lower UA/A ratio.

Table 2. Cox Proportional Hazard Analysis for 30-day mortality

\begin{tabular}{|c|c|c|c|c|}
\hline & \multicolumn{2}{|c|}{ Univariate analysis } & \multicolumn{2}{|c|}{ Multivariate analysis } \\
\hline & P-value & $\mathrm{HR}(95 \% \mathrm{Cl})$ & P-value & $\operatorname{HR}(95 \% \mathrm{Cl})$ \\
\hline Age & 0.029 & $1.03(1.00-1.06)$ & 0.747 & $0.99(0.95-1.03)$ \\
\hline Malignancy & 0.029 & $1.96(1.07-3.58)$ & 0.373 & $1.44(0.65-3.19)$ \\
\hline Carlson comorbidity score & 0.001 & $1.22(1.08-1.38)$ & 0.169 & $1.14(0.95-1.37)$ \\
\hline Respiratory rate & 0.000 & $1.11(1.06-1.16)$ & 0.033 & $1.07(1.01-1.14)$ \\
\hline Ascides & 0.012 & $2.39(1.22-4.71)$ & 0.279 & $1.72(0.64-4.59)$ \\
\hline Pretibial edema & 0.084 & $1.73(0.93-3.22)$ & & \\
\hline Corrected calcium & 0.060 & 1.19 (1.04-1.35) & & \\
\hline Albumin & 0.003 & 0.93 (0.89-0.98) & & \\
\hline Uric acid & 0.019 & $1.11(1.02-1.21)$ & & \\
\hline UA/A ratio & 0.000 & $1.45(1.18-1.80)$ & 0.029 & $1.39(1.02-1.91)$ \\
\hline Power of Hydrogen & 0.058 & $37.9(0.89-1625)$ & & \\
\hline Proteinuria dipstick & 0.010 & 0.57 (0.37-0.87) & & \\
\hline Hypoalbuminemia & 0.033 & $2.16(1.06-4.37)$ & & \\
\hline Hyperuricemia & 0.055 & $1.95(0.98-3.88)$ & & \\
\hline High UA/A ratio & 0.003 & $2.94(1.45-5.96)$ & & \\
\hline
\end{tabular}

$\mathrm{HR}$, hazard ratio; $\mathrm{Cl}$, confidential interval; $\mathrm{UA} / \mathrm{A}$, uric acid albumin ratio. 
Table 3. Comparative characteristics of patients according to UA/A ratio

\begin{tabular}{|c|c|c|c|}
\hline & $\begin{array}{c}\text { UA/A ratio } \\
\quad<2.36 \\
(n=71)\end{array}$ & $\begin{array}{c}\text { UA/A ratio } \\
>2.36 \\
(n=91)\end{array}$ & P-value \\
\hline \multicolumn{4}{|l|}{ Demographics and comorbidities } \\
\hline Age (yr) & $68.5(12.2)$ & $70.1(13.3)$ & 0.434 \\
\hline Sex, female & $32(45.1)$ & $44(48.4)$ & 0.678 \\
\hline Diabetes mellitus & $22(31.0)$ & $37(40.7)$ & 0.204 \\
\hline Duration of diabetes (yr) & $11.3(6.0)$ & $15.5(8.1)$ & 0.087 \\
\hline Hypertension & $44(62.0)$ & $58(63.7)$ & 0.818 \\
\hline Duration of hypertension (yr) & $11.2(8.7)$ & $14.0(9.2)$ & 0.164 \\
\hline Chronic kidney disease & $42(59.2)$ & $41(45.1)$ & 0.075 \\
\hline Duration of chronic kidney disease (yr) & $5.5(8.3)$ & $5.9(5.2)$ & 0.837 \\
\hline Liver cirrhosis & $0(0.0)$ & $6(6.6)$ & 0.029 \\
\hline Duration of cirrhosis (yr) & & $1.0(1.2)$ & \\
\hline Oncological malignancy & $19(26.8)$ & $32(35.2)$ & 0.253 \\
\hline Duration of oncological malignancy (yr) & $3.6(4.5)$ & $3.7(5.0)$ & 0.982 \\
\hline Charlson comorbidity score & $4.8(2.5)$ & $5.8(2.4)$ & 0.007 \\
\hline \multicolumn{4}{|l|}{ Clinical and laboratory findings } \\
\hline Systolic blood pressure (mmHg) & $126.9(29.3)$ & $117.9(27.2)$ & 0.049 \\
\hline Diastolic blood pressure $(\mathrm{mmHg})$ & $74.5(13.3)$ & $69.3(15.3)$ & 0.026 \\
\hline Pulse rate (bpm/min) & $85.8(18.2)$ & $88.5(18.3)$ & 0.361 \\
\hline Oxygen saturation (\%) & $95.2(2.6)$ & $94.1(4.2)$ & 0.174 \\
\hline Body temperature $\left({ }^{\circ} \mathrm{C}\right)$ & $36.8(0.6)$ & $36.8(0.6)$ & 0.896 \\
\hline Respiratory rate (rpm/min) & $15.2(5.2)$ & $16.7(5.2)$ & 0.092 \\
\hline Ascites (\%) & $5(8.1)$ & $20(23.8)$ & 0.013 \\
\hline Pretibial edema (\%) & $19(28.4)$ & $44(51.2)$ & 0.008 \\
\hline Glucose (mg/dL) & $137.0(55.9)$ & $152.2(121.6)$ & 0.337 \\
\hline Basal creatinine (mg/dL) & $1.5(0.9)$ & $1.5(1.0)$ & 0.858 \\
\hline Creatinine (mg/dL) & $4.2(2.0)$ & $4.0(2.3)$ & 0.615 \\
\hline Urea (mg/dL) & $148.5(64.0)$ & $171.5(71.8)$ & 0.035 \\
\hline Total protein (mg/dL) & $63.7(11.0)$ & $61.5(10.1)$ & 0.270 \\
\hline Albumin (mg/dL) & $34.8(6.8)$ & $30.2(6.2)$ & 0.000 \\
\hline Uric acid (mg/dL) & $5.8(1.6)$ & $10.3(2,7)$ & 0.000 \\
\hline UACR (mg/g) & $1,155.3(1,166.2)$ & $602.5(624.0)$ & 0.155 \\
\hline Sodium (mEq/L) & $135.0(6.6)$ & $132.8(7.3)$ & 0.048 \\
\hline Potassium (mEq/L) & $4.8(1.1)$ & $5.0(1.0)$ & 0.358 \\
\hline Chlorine (mEq/L) & $103.2(7.4)$ & $100.4(7.8)$ & 0.021 \\
\hline Calcium (mg/dL) & $8.5(1.1)$ & $8.8(1.9)$ & 0.217 \\
\hline Corrected calcium (mg/dL) & $8.9(1.1)$ & $9.6(1.8)$ & 0.005 \\
\hline Phosphorusm (mg/dL) & $4.2(1.5)$ & $4.6(1.6)$ & 0.103 \\
\hline Hemoglobin (g/dL) & $9.6(2.3)$ & $10.1(2.4)$ & 0.231 \\
\hline Power of hydrogen & $7.3(0.1)$ & $7.3(0.1)$ & 0.420 \\
\hline Bicarbonate (mEq/L) & $18.7(4.6)$ & $19.3(5.1)$ & 0.388 \\
\hline \multicolumn{4}{|l|}{ Outcomes } \\
\hline Length of hospital stay) & $12.3(10.3)$ & $11.6(11.1)$ & 0.704 \\
\hline Length of hospital stay ${ }^{b)}$ & $12.9(10.8)$ & $14.2(11.9)$ & 0.476 \\
\hline 30-Day survival time & $27.7(6.6)$ & $24.0(9.6)$ & 0.006 \\
\hline In-hospital mortality rate & $10(14.1)$ & $29(31.9)$ & 0.009 \\
\hline 30-Day mortality rate & $10(14.1)$ & $33(36.3)$ & 0.002 \\
\hline
\end{tabular}

Values are presented as median (interquartile range) or number (\%). $U A / A$, uric acid albumin ratio; $U A C R$, urine albumin creatinine ratio.

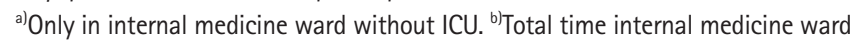
with intensive care unit.

\section{DISCUSSION}

This study demonstrated the relationship between UA/A ratio on initial admission in patients with AKI and the 30-day mortality rate. It has been shown that the relationship between mortality and UA/A ratio exists independent of age, malignancy, Charlson comorbidity score, respiratory rate, and presence of ascites in multivariate models. Also, in this study, the best UA/A ratio cutoff level associated with 30-day mortality was determined as 2.36 $\mathrm{mg} / \mathrm{g}$ in patients with AKI (sensitivity, 77\%; specificity, 52\%; AUROC, 0.644; DOR, 3.59; J, 28.8).

In a study conducted by Yeter et al., ${ }^{12}$ UA/A ratio was found to be associated only with the development of AKI, and its relation with mortality could not be determined. We determined that the UA/A ratio is associated with an increased 30-day mortality risk in patients who develop AKI in this study. It has long been known that hypoalbuminemia is an independent predictor for mortality and morbidity. Shao et al. ${ }^{10}$ found that hypoalbuminemia is an independent risk factor for AKI development and long-term mortality. In a meta-analysis compiled by Wiedermann et al.., ${ }^{14}$ hypoalbuminemia was shown to be predictive of AKI development and mortality post-AKI. In our study, we could not mention any risk factors in the development of AKI since we included patients who had already developed AKI at our starting point. However, hypoalbuminemia has been associated with increased mortality in patients with AKI. It should also be noted that the albumin levels of patients with noted mortality at the 30-day follow-up were significantly lower compared to the survivors during their hospital admission. A possible explanation is a malnutrition state, which can directly affect serum albumin levels. ${ }^{15}$ However, it should be noted that no evidence has been found supporting albumin replacement to treat hypoalbuminemia in order to increase survivability rates. ${ }^{16}$

In patients with DM, it was detected that serum uric acid levels correlated with urinary albumin excretion, ${ }^{16}$ and this was associated with microalbuminuria and subclinical atherosclerosis. ${ }^{17}$ Uric acid levels were also shown to be a possible biomarker in the development of diabetic nephropathy. ${ }^{18}$ It is known that urinary albumin excretion is one of the causes of hypoalbuminemia in both CKD and AKI patients. ${ }^{14,19,20}$ It is also known that albuminuria increases the risk of cardiovascular and all-cause mortality, even in the general population. ${ }^{21} \mathrm{~A}$ striking point in our study was the difference in serum albumin levels, though there was no difference between the urinary albumin excretion rates of both groups according to the 30-day mortality status and UA/A ratio. This suggests that the cause of hypoalbuminemia development is as a negative acute phase reactant rather than via urinary loss. Charl- 
son comorbidity scores, which we found higher in patients with high UA/A ratio compared to those with low ratios; and also, in non-survivors than survivors, provides evidence for this opinion.

A positive relationship has been reported between hyperuricemia and cardiovascular disease, but no study has yet proven the definitive role of serum uric acid in the development of cardiovascular disease. In addition, although the antioxidant effect of uric acid has recently been shown to inhibit DNA damage, no epidemiological studies have documented reduced cancer risk among people with hyperuricemia. Tomita et al. ${ }^{22}$ reported in a 5-year follow-up study on about fifty thousand workers that hyperuricemia has been shown to be strongly associated with all-cause deaths, coronary artery disease, stroke, liver disease, and renal failure. In our study, although there were higher uric acid levels in the nonsurvivor group, we could not find a relationship between 30-day mortality and hyperuricemia. This may be because our follow-up time was considerably shorter than other recent studies that have demonstrated the relationship of hyperuricemia with long-term mortality.

Previous studies have shown that both low albumin and high uric acid levels are associated with development of AKI and increased mortality. By combining these two strongly related situations seen in the literature, we have created a stronger predictor of mortality. In short-term mortality estimations, we determined the best sensitivity and specificity rates, AUROC, DOR and J as well as the best cut-off level. When we divide our patients into two groups according to this cut-of level, it was also emphasized that high UA/A ratio was significantly associated with high mortality rate and low survival time.

Despite the strengths of our study, there were some limitations. First of all, patient treatments during hospitalization and medical conditions that developed after hospitalization, which were thought to have an effect on mortality, were not included in the study. This was because our study was designed based on only initial data recorded in the hospital admission. Secondly, our study cannot be generalized for other disease groups since it was performed in the AKI patients group only. In spite of this, it can provide a template for the studies to be carried out with different disease groups and for wider populations.

In summary, our study population consisted of renal, prerenal and postrenal AKI patients whose disease was caused by many different pathophysiological mechanisms such as hepatorenal syndrome due to liver cirrhosis, chemotherapy toxicity, contrast nephropathy, dehydration, and bladder cancer. We aimed to investigate the relationship between mortality rates and the synergism of both hyperuricemia and hypoalbuminemia independent of these etiological factors. Indeed, regardless of age, Charlson comorbidity score, and clinical parameters, we found the above mentioned to be associated with an increased risk of short-term mortality. We thus present a result-oriented practical approximate without etiological differences.

\section{CONFLICT OF INTEREST}

No potential conflict of interest relevant to this article was reported.

\section{REFERENCES}

1. Fanali G, di Masi A, Trezza V, Marino M, Fasano M, Ascenzi P. Human serum albumin: from bench to bedside. Mol Aspects Med 2012;33:209-90.

2. Roche $M$, Rondeau $P$, Singh NR, Tarnus E, Bourdon E. The antioxidant properties of serum albumin. FEBS Lett 2008;582: 1783-7.

3. Peters T Jr. Serum albumin. Adv Protein Chem 1985;37:161245.

4. Fleck $A$, Raines $G$, Hawker $F$, et al. Increased vascular permeability: a major cause of hypoalbuminaemia in disease and injury. Lancet 1985;1:781-4.

5. Vincent JL, Russell JA, Jacob M, et al. Albumin administration in the acutely ill: what is new and where next? Crit Care 2014; 18:231.

6. Tanabe Y. Studies on uric acid metabolism in man. I. Uric acid metabolism in man studied by means of $14 \mathrm{C}$-labeled uric acid. Naika Hokan 1971;18:371-7.

7. Zhu Y, Pandya BJ, Choi HK. Prevalence of gout and hyperuricemia in the US general population: the National Health and Nutrition Examination Survey 2007-2008. Arthritis Rheum 2011:63:3136-41.

8. Abdelmalek MF, Diehl AM. Nonalcoholic fatty liver disease as a complication of insulin resistance. Med Clin North Am 2007; 91:1125-49.

9. Ronco C, Bellomo R, Kellum JA. Acute kidney injury. Lancet 2019;394:1949-64.

10. Shao $M$, Wang $S$, Parameswaran PK. Hypoalbuminemia: a risk factor for acute kidney injury development and progression to chronic kidney disease in critically ill patients. Int Urol Nephrol 2017:49:295-302.

11. Kim JH, Gil HW, Yang JO, Lee EY, Hong SY. Serum uric acid level as a marker for mortality and acute kidney injury in patients with acute paraquat intoxication. Nephrol Dial Transplant 2011;26:1846-52.

12. Yeter HH, Eyupoglu D, Pasayev T, Cetik S, Akcay OF, Yildirim T. 
Role of uric acid albumin ratio in predicting development of acute kidney injury and mortality in intensive care unit patients. Turkish J Nephrol 2019;28:160-7.

13. Khwaja A. KDIGO clinical practice guidelines for acute kidney injury. Nephron Clin Pract 2012;120:c179-84.

14. Wiedermann CJ, Wiedermann W, Joannidis M. Hypoalbuminemia and acute kidney injury: a meta-analysis of observational clinical studies. Intensive Care Med 2010;36:1657-65.

15. Don BR, Kaysen G. Serum albumin: relationship to inflammation and nutrition. Semin Dial 2004;17:432-7.

16. Tseng $\mathrm{CH}$. Correlation of uric acid and urinary albumin excretion rate in patients with type 2 diabetes mellitus in Taiwan. Kidney Int 2005;68:796-801.

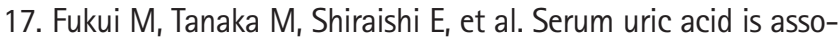
ciated with microalbuminuria and subclinical atherosclerosis in men with type 2 diabetes mellitus. Metabolism 2008;57: 625-9.

18. Hovind P, Rossing P, Tarnow L, Johnson RJ, Parving HH. Serum uric acid as a predictor for development of diabetic nephropathy in type 1 diabetes: an inception cohort study. Diabetes 2009;58:1668-71.

19. Chen TK, Knicely DH, Grams ME. Chronic kidney disease diagnosis and management: a review. JAMA 2019;322:1294-304.

20. James MT, Grams ME, Woodward M, et al. A meta-analysis of the association of estimated GFR, albuminuria, diabetes mellitus, and hypertension with acute kidney injury. Am J Kidney Dis 2015;66:602-12.

21. Chronic Kidney Disease Prognosis Consortium, Matsushita K, van der Velde $\mathrm{M}$, et al. Association of estimated glomerular filtration rate and albuminuria with all-cause and cardiovascular mortality in general population cohorts: a collaborative meta-analysis. Lancet 2010;375:2073-81.

22. Tomita M, Mizuno S, Yamanaka $H$, et al. Does hyperuricemia affect mortality? A prospective cohort study of Japanese male workers. J Epidemiol 2000;10:403-9. 